\title{
QUASI-GREEN'S FUNCTION APPROACH TO FUNDAMENTAL FREQUENCY ANALYSIS OF ELASTICALLY SUPPORTED THIN CIRCULAR AND ANNULAR PLATES WITH ELASTIC CONSTRAINTS
}

\author{
KRZYSZTOF K. ŻuR \\ Bialystok University of Technology, Faculty of Management, Kleosin, Poland \\ e-mail:k.zur@pb.edu.pl
}

\begin{abstract}
Free vibration analysis of homogeneous and isotropic thin circular and annular plates with discrete elements such as elastic ring supports is considered. The general form of quasi-Green's function for thin circular and annular plates is obtained. The nonlinear characteristic equations are defined for thin circular and annular plates with different boundary conditions and different combinations of the core and support radius. The continuity conditions at the ring supports are omitted based on the properties of Green's function. The fundamental frequency of axisymmetric vibration has been calculated using the Newton-Raphson method and calculation software. The obtained results are compared with selected results presented in literature. The exact frequencies of vibration presented in a non-dimensional form can serve as benchmark values for researchers to validate their numerical methods when applied for uniform thin circular and annular plate problems.
\end{abstract}

Keywords: quasi-Green's function, ring supports, movable edges, elastic constraints

\section{Introduction}

The study of vibration of a thin circular and annular plate is basic in structural mechanics. Components of circular and annular plates are commonly used in the aerospace industry and aviation as well as in marine and civil engineering applications. Circular and annular plates are the most critical structural elements in high speed rotating engineering systems. The natural frequencies of circular and annular plates have been studied extensively for more than a century, because if only the frequency of external load matches the natural frequency of the plate, destruction may occur. Additionally, the influence of elastic or rigid ring supports on dynamic behavior of plates have been studied in a lot of works, because it used to stabilize or to increase the frequency of plates. Knowledge about distribution of ring supports of variable stiffness can allow one to predict dynamic behavior of structural elements such us circular and annular plates.

The free vibration of circular and annular plates with concentric ring supports have been studied in a lot of works. Bodine (1967) studied the influence of rigid supports on the fundamental frequency of circular plates in which radius of the supports was small. Kunukkasseril and Swamidas (1974) formulated equations for circular plates with elastic supports, but they solved the free vibration problem for a free circular plate. Singh and Mirza (1976) studied free axisymmetric vibration of circular plates elastically supported along two concentric circles. Azimi (1988) studied natural vibration of circular plates with elastic and rigid supports using the receptance method. Wang and Thevendran (1993) analyzed free vibration of annular plates with concentric supports using by the Rayleigh-Ritz method. Ding (1994) solved the free vibration problem for arbitrarily shaped plates with concentric elastic and rigid ring supports. Liu and Chen (1995) studied axisymmetric vibration of annular and circular plates using simple finite analysis. In works by Vega et al. (1999) free vibration analysis was presented for a concentrically 
supported annular plate with a free edge using the optimized Rayleigh-Ritz method. Laura et al. (1999) analyzed transverse vibration of a circular plate with a free edge and concentric ring supports. Vega et al. (2000) analyzed free vibration of concentrically supported annular plates with one edge clamped or simply supported. The fundamental frequency of a free thin circular plate supported on a ring was analyzed by Wang (2001). Influence of the stiffness and location of elastic ring supports on the fundamental frequency of circular plates were analyzed by Wang and Wang (2003). Wang (2006, 2014) studied vibration modes of concentrically supported free circular and annular plates with movable edges. Rao and Rao (2014a) analyzed free vibration of annular plates with both edges elastically restrained and resting on the Winkler foundation. Additionally, Rao and Rao (2014b) analyzed free vibration of a thin circular plate with concentric ring and elastic edge support.

In the works presented above, the analyzed plates were separated into two regions for one ring supports. The number of separated regions increases if the number of considered elastic ring supports increases. In this approach, the solution to boundary value problem is complicated. Additionally, continuity conditions between the support and plate must be used to obtain characteristic equations. Solution to the boundary value problem is very tedious and more complicated based on continuity conditions, because characteristic matrices have a large dimension.

Application of Green's function to the solution to the boundary value problem of free vibration of plates allow one to neglect the continuity condition. In the works of Kukla and Szewczyk (2004, 2005, 2007) Green's function approach to frequency analysis is presented for circular and annular thin plates with elastic supports. The authors calculated nontrivial constants of general solutions to the differential equation to obtain a full form of Green's function for free, simply-supported and clamped plates. The nontrivial constants have a very complicated form, and calculating them is very tedious for different boundary conditions such as sliding supports or elastic constraints.

The novelty of the paper is quasi-Green's function (not full form) approach to obtain characteristic equations of concentrically supported circular and annular plates with clamped, free, simply-supported and sliding (movable) edges or elastic constraints. The quasi-Green function is obtained by the method presented in the previous works (Żur, 2015, 2016a). Nonlinear characteristic equations of plates are obtained without calculating nontrivial constants of the general solution to the differential equation. The numerical results of investigation are compared with selected results presented in literature. The exact fundamental frequencies of axisymmetric vibration are presented in a non-dimensional form for different combinations of the core and support radius as well as selected values of parameters of elastic constraints.

\section{Statement of the problem}

Consider an isotropic, homogeneous annular (circular) thin plate of constant thickness $\mathrm{h}$ in cylindrical coordinates $(r, \theta, z)$ with the $z$-axis along the longitudinal direction. The geometry and coordinate system of the considered plate is shown in Fig. 1. The partial differential equation for free vibration of thin uniform annular (circular) plates has the following form

$$
\nabla^{4} W(r, t)+\frac{\rho h}{D} \frac{\partial^{2} W(r, t)}{\partial t^{2}}=-\sum_{j=1}^{\chi} K_{j} W(r, t) \delta\left(r-r_{j}\right)
$$

where $\rho$ is mass density, $D=E h^{3} /\left[12\left(1-\nu^{2}\right)\right]$ is flexural rigidity, $E$ is Young's modulus, $\nu$ is Poisson's ratio, $\nabla^{2}=\left(\partial^{2} / \partial r^{2}\right)+(1 / r)(\partial / \partial r)$ is Laplacian, $K_{j}$ is a coefficient of normalized stiffness of the supports, $\delta$ is Dirac's delta function, $r_{j}$ is the position of elastic ring supports, $\chi$ is the number of elastic ring supports and $W(r, t)$ is small deflection compared with thickness $h$ of the plate. 


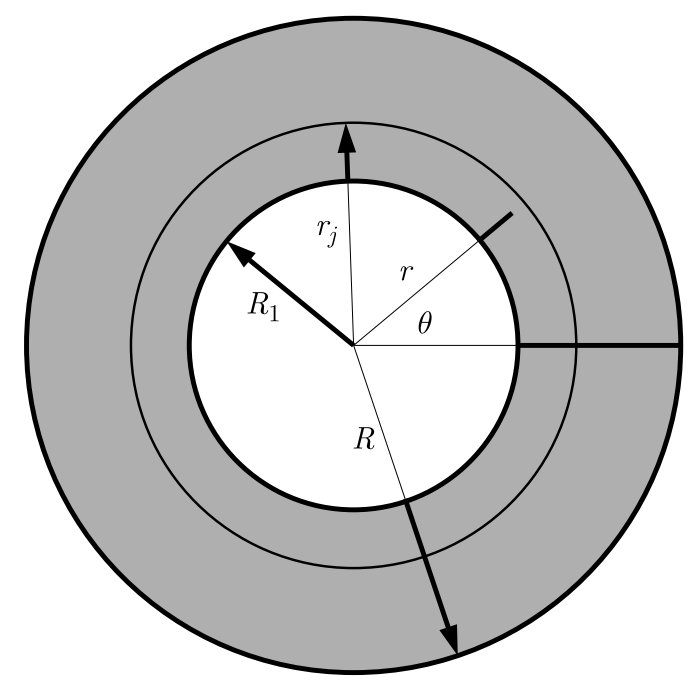

Fig. 1. The geometry and coordinate system of the annular plate with radius of the hole $R_{1}$

The axisymmetric deflection of an annular (circular) plate may be expressed as follows

$$
W(r, t)=w(r) \mathrm{e}^{\mathrm{i} \omega t}
$$

where $w(r)$ is the radial mode function, $\omega$ is the natural frequency, and $\mathrm{i}^{2}=-1$. Substituting Eq. (2.2) into Eq. (2.1) and using the dimensionless coordinates $\xi=r / R$ and $\kappa_{j}=r_{j} / R$, the governing differential equation of the annular (circular) plate is obtained

$$
L(w)-\lambda^{2} w=-\sum_{j=1}^{\chi} K_{j} w\left(\kappa_{j}\right) \delta\left(\xi-\kappa_{j}\right)
$$

where

$$
L(w) \equiv \frac{d^{4} w}{d \xi^{4}}+\frac{2}{\xi} \frac{d^{3} w}{d \xi^{3}}-\frac{1}{\xi^{2}} \frac{d^{2} w}{d \xi^{2}}+\frac{1}{\xi^{3}} \frac{d w}{d \xi}
$$

is the differential operator and

$$
\lambda=\omega R^{2} \sqrt{\rho h / D}
$$

is the dimensionless frequency of vibration.

The boundary conditions at the outer edge $(\xi=1)$ of the annular (circular) plate may be one of the following: clamped, simply supported, free, sliding supports and elastic supports. These conditions may be written in terms of the radial mode function $w(\xi)$ in the following form:

- clamped

$$
\left.w(\xi)\right|_{\xi=1}=\left.0 \quad \frac{d w}{d \xi}\right|_{\xi=1}=0
$$

— simply supported

$$
\left.w(\xi)\right|_{\xi=1}=\left.0 \quad M(w)\right|_{\xi=1}=\left(\frac{d^{2} w}{d \xi^{2}}+\frac{\nu}{\xi} \frac{d w}{d \xi}\right)_{\xi=1}=0
$$

- free

$$
\left.M(w)\right|_{\xi=1}=\left.0 \quad V(w)\right|_{\xi=1}=\left(\frac{d^{3} w}{d \xi^{3}}+\frac{1}{\xi} \frac{d^{2} w}{d \xi^{2}}-\frac{1}{\xi^{2}} \frac{d w}{d \xi}\right)_{\xi=1}=0
$$


— movable edges (sliding)

$$
\left.\frac{d w}{d \xi}\right|_{\xi=1}=\left.0 \quad V(w)\right|_{\xi=1}=0
$$

- elastic supports

$$
\begin{aligned}
& \left.\Phi(w)\right|_{\xi=1}=\left[\left(\frac{d^{2} w}{d \xi^{2}}+\nu \frac{d w}{d \xi}\right)+\phi \frac{d w}{d \xi}\right]_{\xi=1}=0 \\
& \left.\Psi(w)\right|_{\xi=1}=\left[\left(\frac{d^{3} w}{d \xi^{3}}+\frac{d^{2} w}{d \xi^{2}}-\frac{d w}{d \xi}\right)-\psi w\right]_{\xi=1}=0
\end{aligned}
$$

$M(w)$ and $V(w)$ are the normalized radial bending moment and the normalized effective shear force, respectively. $\phi=K_{\phi} R / D_{R}$ and $\psi=K_{\psi} R^{3} / D_{R}$ are the parameters of elastic constraints. $K_{\phi}$ and $K_{\psi}$ are the rotational and translational spring constants, respectively. Similar boundary conditions may be defined at the inner edge $\left(\xi=R_{1} / R=\xi_{1}\right)$, depending on considered annular plates.

\section{Finding quasi-Green's function}

The general solution to the homogeneous differential equation for thin annular (circular) plates

$$
L(w)-\lambda^{2} w=0
$$

is a linear combination of the Bessel functions presented in the following form (McLachlan, 1955)

$$
w(\xi)=C_{1} J_{0}(\lambda \xi)+C_{2} I_{0}(\lambda \xi)+C_{3} Y_{0}(\lambda \xi)+C_{4} K_{0}(\lambda \xi)
$$

where $J_{0}(\lambda \xi), Y_{0}(\lambda \xi)$ are the Bessel functions of the first and second kind, $I_{0}(\lambda \xi), K_{0}(\lambda \xi)$ are the modified Bessel functions of the first and second kind. The quasi-Green function $K(\xi, \alpha)$ is a particular solution to Eq. (3.1) and may be received from the formula presented in the following form (Jaroszewicz and Zoryj, 2005; Żur, 2015)

$$
K(\xi, \alpha)=\frac{D(\xi, \alpha)}{W(\alpha) p_{0}(\alpha)}
$$

where $p_{0}(\alpha)=1$ is a coefficient placed in front of the highest order of derivative of differential equation (3.1), and

$$
\begin{gathered}
D(\xi, \alpha)=\left|\begin{array}{cccc}
J_{0}(\lambda \alpha) & I_{0}(\lambda \alpha) & Y_{0}(\lambda \alpha) & K_{0}(\lambda \alpha) \\
\frac{d J_{0}(\lambda \alpha)}{d \alpha} & \frac{d I_{0}(\lambda \alpha)}{d \alpha} & \frac{d Y_{0}(\lambda \alpha)}{d \alpha} & \frac{d K_{0}(\lambda \alpha)}{d \alpha} \\
\frac{d^{2} J_{0}(\lambda \alpha)}{d \alpha^{2}} & \frac{d^{2} I_{0}(\lambda \alpha)}{d \alpha^{2}} & \frac{d^{2} Y_{0}(\lambda \alpha)}{d \alpha^{2}} & \frac{d^{2} K_{0}(\lambda \alpha)}{d \alpha^{2}} \\
J_{0}(\lambda \xi) & I_{0}(\lambda \xi) & Y_{0}(\lambda \xi) & K_{0}(\lambda \xi)
\end{array}\right| \\
W(\alpha)=\left|\begin{array}{cccc}
J_{0}(\lambda \alpha) & I_{0}(\lambda \alpha) & Y_{0}(\lambda \alpha) & K_{0}(\lambda \alpha) \\
\frac{d J_{0}(\lambda \alpha)}{d \alpha} & \frac{d I_{0}(\lambda \alpha)}{d \alpha} & \frac{d Y_{0}(\lambda \alpha)}{d \alpha} & \frac{d K_{0}(\lambda \alpha)}{d \alpha} \\
\frac{d^{2} J_{0}(\lambda \alpha)}{d \alpha^{2}} & \frac{d^{2} I_{0}(\lambda \alpha)}{d \alpha^{2}} & \frac{d^{2} Y_{0}(\lambda \alpha)}{d \alpha^{2}} & \frac{d^{2} K_{0}(\lambda \alpha)}{d \alpha^{2}} \\
\frac{d^{3} J_{0}(\lambda \alpha)}{d \alpha^{3}} & \frac{d^{3} I_{0}(\lambda \alpha)}{d \alpha^{3}} & \frac{d^{3} Y_{0}(\lambda \alpha)}{d \alpha^{3}} & \frac{d^{3} K_{0}(\lambda \alpha)}{d \alpha^{3}}
\end{array}\right|
\end{gathered}
$$


The elements of the matrix $\mathbf{D}$ and $\mathbf{W}$ have the following form

$$
\begin{array}{llrl}
\frac{d J_{0}(\lambda \alpha)}{d \alpha} & =-\lambda J_{1}(\lambda \alpha) & \frac{d I_{0}(\lambda \alpha)}{d \alpha}=\lambda I_{1}(\lambda \alpha) & \\
\frac{d Y_{0}(\lambda \alpha)}{d \alpha} & =-\lambda Y_{1}(\lambda \alpha) & \frac{d K_{0}(\lambda \alpha)}{d \alpha} & = \\
\frac{d^{2} J_{0}(\lambda \alpha)}{d \alpha^{2}} & =\frac{\lambda^{2}}{2}\left[J_{0}(\lambda \alpha)+K_{1}(\lambda \alpha)\right] & \frac{d^{2} I_{0}(\lambda \alpha)}{d \alpha^{2}}=\frac{\lambda^{2}}{2}\left[I_{0}(\lambda \alpha)+I_{2}(\lambda \alpha)\right] \\
\frac{d^{2} Y_{0}(\lambda \alpha)}{d \alpha^{2}}=\frac{\lambda^{2}}{2}\left[Y_{0}(\lambda \alpha)+Y_{2}(\lambda \alpha)\right] & \frac{d^{2} K_{0}(\lambda \alpha)}{d \alpha^{2}}=\frac{\lambda^{2}}{2}\left[K_{0}(\lambda \alpha)+K_{2}(\lambda \alpha)\right] \\
\frac{d^{3} J_{0}(\lambda \alpha)}{d \alpha^{3}}=\frac{\lambda^{3}}{4}\left[3 J_{1}(\lambda \alpha)+J_{3}(\lambda \alpha)\right] & \frac{d^{3} I_{0}(\lambda \alpha)}{d \alpha^{3}}=\frac{\lambda^{3}}{4}\left[3 I_{1}(\lambda \alpha)+I_{3}(\lambda \alpha)\right] \\
\frac{d^{3} Y_{0}(\lambda \alpha)}{d \alpha^{3}}=\frac{\lambda^{3}}{4}\left[3 Y_{1}(\lambda \alpha)-Y_{3}(\lambda \alpha)\right] & \frac{d^{3} K_{0}(\lambda \alpha)}{d \alpha^{3}}=-\frac{\lambda^{3}}{4}\left[3 K_{1}(\lambda \alpha)+K_{3}(\lambda \alpha)\right]
\end{array}
$$

After calculations, the function $D(\xi, \alpha)$ has the form

$$
D(\xi, \alpha)=\frac{2 \lambda^{2}}{\pi \alpha}\left[2 I_{0}(\lambda \xi) K_{0}(\lambda \alpha)-2 I_{0}(\lambda \alpha) K_{0}(\lambda \xi)+\pi J_{0}(\lambda \xi) Y_{0}(\lambda \alpha)-\pi J_{0}(\lambda \alpha) Y_{0}(\lambda \xi)\right]
$$

Bessel function (3.2) expresses linear independent solutions, thus the Wronskian must satisfy the condition (Stakgold and Holst, 2011)

$$
W(\alpha)=\frac{8 \lambda^{4}}{\pi \alpha^{2}} \neq 0
$$

Condition (3.9) is satisfied for a circular plate $(0<\alpha \leqslant 1)$ and an annular plate $\left(0<\xi_{1} \leqslant \alpha \leqslant 1\right)$.

After calculations, the quasi-Green function has the form

$$
K(\xi, \alpha)=\frac{\alpha}{4 \lambda^{2}}\left[2 I_{0}(\lambda \xi) K_{0}(\lambda \alpha)-2 I_{0}(\lambda \alpha) K_{0}(\lambda \xi)-\pi J_{0}(\lambda \alpha) Y_{0}(\lambda \xi)+\pi J_{0}(\lambda \xi) Y_{0}(\lambda \alpha)\right]
$$

and satisfies the conditions

$$
K(a, a)=\left.\frac{\partial K(\xi, \alpha)}{\partial \xi}\right|_{\xi=a}=\left.\frac{\partial^{2} K(\xi, \alpha)}{\partial \xi^{2}}\right|_{\xi=a}=\left.0 \quad \frac{\partial^{3} K(\xi, \alpha)}{\partial \xi^{3}}\right|_{\xi=a}=1
$$

according to properties of the influence functions (Stakgold and Holst, 2011).

\section{Solution of the problem for the circular plate}

In the previous paper (Żur, 2016b), the possibility of solving the similar boundary value problem was proposed for non-uniform annular plates without calculations. Based on the paper of $\dot{Z} u r$

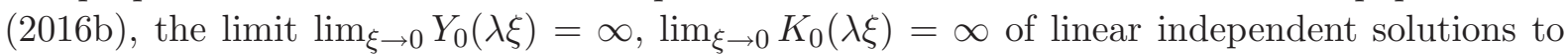
Eq. (2.3) for the circular plate can be presented in the following form

$$
\begin{aligned}
K(\xi, \lambda, \boldsymbol{\kappa}, \mathbf{K})_{a} & =J_{0}(\lambda \xi)-\sum_{j=1}^{\chi} K_{j} J_{0}\left(\lambda \kappa_{j}\right) G\left(\xi, \kappa_{j}\right) \\
K(\xi, \lambda, \boldsymbol{\kappa}, \mathbf{K})_{b} & =I_{0}(\lambda \xi)-\sum_{j=1}^{\chi} K_{j} I_{0}\left(\lambda \kappa_{j}\right) G\left(\xi, \kappa_{j}\right)
\end{aligned}
$$


where

$$
\begin{aligned}
& G\left(\xi, \kappa_{j}\right)=K\left(\xi, \kappa_{j}\right) H\left(\xi-\kappa_{j}\right) \\
& K\left(\xi, \kappa_{j}\right)=\frac{\kappa_{j}}{4 \lambda^{2}}\left[2 I_{0}(\lambda \xi) K_{0}\left(\lambda \kappa_{j}\right)-2 I_{0}\left(\lambda \kappa_{j}\right) K_{0}(\lambda \xi)-\pi J_{0}\left(\lambda \kappa_{j}\right) Y_{0}(\lambda \xi)+\pi J_{0}(\lambda \xi) Y_{0}\left(\lambda \kappa_{j}\right)\right]
\end{aligned}
$$

and

$$
\boldsymbol{\kappa}=\left[\kappa_{1}, \ldots, \kappa_{\chi}\right] \quad \mathbf{K}=\left[K_{1}, \ldots, K_{\chi}\right]
$$

and $H\left(\xi-\kappa_{j}\right)$ is the Heaviside function.

The characteristic equations $\Delta=0$ of the circular plate for different boundary conditions and different values of the parameters $\kappa_{j}$ and $K_{j}$ are obtained from well known characteristic determinants given by:

- clamped

$$
\left.\Delta(\lambda, \boldsymbol{\kappa}, \mathbf{K}) \equiv\left|\begin{array}{cc}
K(\xi, \lambda, \boldsymbol{\kappa}, \mathbf{K})_{a} & K(\xi, \lambda, \boldsymbol{\kappa}, \mathbf{K})_{b} \\
\frac{\partial K(\xi, \lambda, \boldsymbol{\kappa}, \mathbf{K})_{a}}{\partial \xi} & \frac{\partial K(\xi, \lambda, \boldsymbol{\kappa}, \mathbf{K})_{b}}{\partial \xi}
\end{array}\right|\right|_{\xi=1}
$$

— simply supported

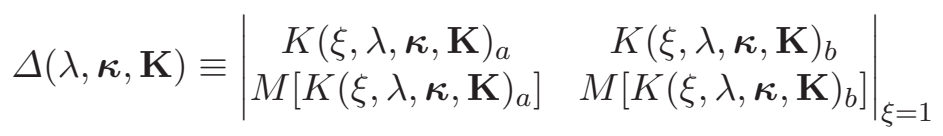

- free

$$
\Delta(\lambda, \boldsymbol{\kappa}, \mathbf{K}) \equiv\left|\begin{array}{ll}
M\left[K(\xi, \lambda, \boldsymbol{\kappa}, \mathbf{K})_{a}\right] & M\left[K(\xi, \lambda, \boldsymbol{\kappa}, \mathbf{K})_{b}\right] \\
V\left[K(\xi, \lambda, \boldsymbol{\kappa}, \mathbf{K})_{a}\right] & V\left[K(\xi, \lambda, \boldsymbol{\kappa}, \mathbf{K})_{b}\right]
\end{array}\right|_{\xi=1}
$$

— sliding

$$
\Delta(\lambda, \boldsymbol{\kappa}, \mathbf{K}) \equiv\left|\begin{array}{lc}
\frac{\partial K(\xi, \lambda, \boldsymbol{\kappa}, \mathbf{K})_{a}}{\partial \xi} & \frac{\partial K(\xi, \lambda, \boldsymbol{\kappa}, \mathbf{K})_{b}}{\partial \xi} \\
V\left[K(\xi, \lambda, \boldsymbol{\kappa}, \mathbf{K})_{a}\right] & V\left[K(\xi, \lambda, \boldsymbol{\kappa}, \mathbf{K})_{b}\right]
\end{array}\right|_{\xi=1}
$$

- elastic supports

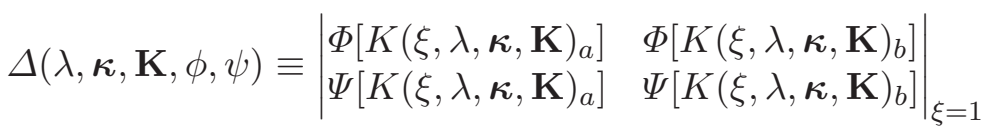

\section{Solution of the problem for the annular plate}

The linear independent solutions to Eq. (2.3) for the annular plate can be presented in the following form

$$
\begin{aligned}
& \mathcal{B}_{a} \equiv K(\xi, \lambda, \boldsymbol{\kappa}, \mathbf{K})_{a}=J_{0}(\lambda \xi)-\sum_{j=1}^{\chi} K_{j} J_{0}\left(\lambda \kappa_{j}\right) G\left(\xi, \kappa_{j}\right) \\
& \mathcal{B}_{b} \equiv K(\xi, \lambda, \boldsymbol{\kappa}, \mathbf{K})_{b}=I_{0}(\lambda \xi)-\sum_{j=1}^{\chi} K_{j} I_{0}\left(\lambda \kappa_{j}\right) G\left(\xi, \kappa_{j}\right) \\
& \mathcal{B}_{c} \equiv K(\xi, \lambda, \boldsymbol{\kappa}, \mathbf{K})_{c}=Y_{0}(\lambda \xi)-\sum_{j=1}^{\chi} K_{j} Y_{0}\left(\lambda \kappa_{j}\right) G\left(\xi, \kappa_{j}\right) \\
& \mathcal{B}_{d} \equiv K(\xi, \lambda, \boldsymbol{\kappa}, \mathbf{K})_{d}=K_{0}(\lambda \xi)-\sum_{j=1}^{\chi} K_{j} K_{0}\left(\lambda \kappa_{j}\right) G\left(\xi, \kappa_{j}\right)
\end{aligned}
$$


The characteristic equations $\Delta=0$ of the annular plate for different boundary conditions and different values of the parameters $\kappa_{j}$ and $K_{j}$ are obtained from well known characteristic determinants given by:

— free outer edge and clamped inner edge

$$
\Delta(\lambda, \boldsymbol{\kappa}, \mathbf{K}) \equiv\left|\begin{array}{cccc}
\left.M\left[\mathcal{B}_{a}\right]\right|_{\xi=1} & \left.M\left[\mathcal{B}_{b}\right]\right|_{\xi=1} & \left.M\left[\mathcal{B}_{c}\right]\right|_{\xi=1} & \left.M\left[\mathcal{B}_{d}\right]\right|_{\xi=1} \\
\left.V\left[\mathcal{B}_{a}\right]\right|_{\xi=1} & \left.V\left[\mathcal{B}_{b}\right]\right|_{\xi=1} & \left.V\left[\mathcal{B}_{c}\right]\right|_{\xi=1} & \left.V\left[\mathcal{B}_{d}\right]\right|_{\xi=1} \\
\left.\mathcal{B}_{a}\right|_{\xi=\xi_{1}} & \left.\mathcal{B}_{b}\right|_{\xi=\xi_{1}} & \left.\mathcal{B}_{c}\right|_{\xi=\xi_{1}} & \left.\mathcal{B}_{d}\right|_{\xi=\xi_{1}} \\
\left.\frac{\partial \mathcal{B}_{a}}{\partial \xi}\right|_{\xi=\xi_{1}} & \left.\frac{\partial \mathcal{B}_{b}}{\partial \xi}\right|_{\xi=\xi_{1}} & \left.\frac{\partial \mathcal{B}_{c}}{\partial \xi}\right|_{\xi=\xi_{1}} & \left.\frac{\partial \mathcal{B}_{d}}{\partial \xi}\right|_{\xi=\xi_{1}}
\end{array}\right|
$$

— free outer edge and simply supported inner edge

$$
\Delta(\lambda, \boldsymbol{\kappa}, \mathbf{K}) \equiv\left|\begin{array}{cccc}
\left(\left.M\left[\mathcal{B}_{a}\right]\right|_{\xi=1}\right. & \left.M\left[\mathcal{B}_{b}\right]\right|_{\xi=1} & \left.M\left[\mathcal{B}_{c}\right]\right|_{\xi=1} & \left.M\left[\mathcal{B}_{d}\right]\right|_{\xi=1} \\
\left.V\left[\mathcal{B}_{a}\right]\right|_{\xi=1} & \left.V\left[\mathcal{B}_{b}\right]\right|_{\xi=1} & \left.V\left[\mathcal{B}_{c}\right]\right|_{\xi=1} & \left.V\left[\mathcal{B}_{d}\right]\right|_{\xi=1} \\
\left.\mathcal{B}_{a}\right|_{\xi=\xi_{1}} & \left.\mathcal{B}_{b}\right|_{\xi=\xi_{1}} & \left.\mathcal{B}_{c}\right|_{\xi=\xi_{1}} & \left.\mathcal{B}_{d}\right|_{\xi=\xi_{1}} \\
\left.M\left[\mathcal{B}_{a}\right]\right|_{\xi=\xi_{1}} & \left.M\left[\mathcal{B}_{b}\right]\right|_{\xi=\xi_{1}} & \left.M\left[\mathcal{B}_{c}\right]\right|_{\xi=\xi_{1}} & \left.M\left[\mathcal{B}_{d}\right]\right|_{\xi=\xi_{1}}
\end{array}\right|
$$

— free both edges

$$
\Delta(\lambda, \boldsymbol{\kappa}, \mathbf{K}) \equiv\left|\begin{array}{cccc}
\left.M\left[\mathcal{B}_{a}\right]\right|_{\xi=1} & \left.M\left[\mathcal{B}_{b}\right]\right|_{\xi=1} & \left.M\left[\mathcal{B}_{c}\right]\right|_{\xi=1} & \left.M\left[\mathcal{B}_{d}\right]\right|_{\xi=1} \\
\left.V\left[\mathcal{B}_{a}\right]\right|_{\xi=1} & \left.V\left[\mathcal{B}_{b}\right]\right|_{\xi=1} & \left.V\left[\mathcal{B}_{c}\right]\right|_{\xi=1} & \left.V\left[\mathcal{B}_{d}\right]\right|_{\xi=1} \\
\left.M\left[\mathcal{B}_{a}\right]\right|_{\xi=\xi_{1}} & \left.M\left[\mathcal{B}_{b}\right]\right|_{\xi=\xi_{1}} & \left.M\left[\mathcal{B}_{c}\right]\right|_{\xi=\xi_{1}} & \left.M\left[\mathcal{B}_{d}\right]\right|_{\xi=\xi_{1}} \\
\left.V\left[\mathcal{B}_{a}\right]\right|_{\xi=\xi_{1}} & \left.V\left[\mathcal{B}_{b}\right]\right|_{\xi=\xi_{1}} & \left.V\left[\mathcal{B}_{c}\right]\right|_{\xi=\xi_{1}} & \left.V\left[\mathcal{B}_{d}\right]\right|_{\xi=\xi_{1}}
\end{array}\right|
$$

— free outer edge and sliding inner edge

$$
\Delta(\lambda, \boldsymbol{\kappa}, \mathbf{K}) \equiv\left|\begin{array}{llll}
\left.M\left[\mathcal{B}_{a}\right]\right|_{\xi=1} & \left.M\left[\mathcal{B}_{b}\right]\right|_{\xi=1} & \left.M\left[\mathcal{B}_{c}\right]\right|_{\xi=1} & \left.M\left[\mathcal{B}_{d}\right]\right|_{\xi=1} \\
\left.V\left[\mathcal{B}_{a}\right]\right|_{\xi=1} & \left.V\left[\mathcal{B}_{b}\right]\right|_{\xi=1} & \left.V\left[\mathcal{B}_{c}\right]\right|_{\xi=1} & \left.V\left[\mathcal{B}_{d}\right]\right|_{\xi=1} \\
\left.\frac{\partial \mathcal{B}_{a}}{\partial \xi}\right|_{\xi=\xi_{1}} & \left.\frac{\partial \mathcal{B}_{b}}{\partial \xi}\right|_{\xi=\xi_{1}} & \left.\frac{\partial \mathcal{B}_{c}}{\partial \xi}\right|_{\xi=\xi_{1}} & \left.\frac{\partial \mathcal{B}_{d}}{\partial \xi}\right|_{\xi=\xi_{1}} \\
\left.V\left[\mathcal{B}_{a}\right]\right|_{\xi=\xi_{1}} & \left.V\left[\mathcal{B}_{b}\right]\right|_{\xi=\xi_{1}} & \left.V\left[\mathcal{B}_{c}\right]\right|_{\xi=\xi_{1}} & \left.V\left[\mathcal{B}_{d}\right]\right|_{\xi=\xi_{1}}
\end{array}\right|
$$

— free inner edge and clamped outer edge

$$
\Delta(\lambda, \boldsymbol{\kappa}, \mathbf{K}) \equiv\left|\begin{array}{cccc}
\left.M\left[\mathcal{B}_{a}\right]\right|_{\xi=\xi_{1}} & \left.M\left[\mathcal{B}_{b}\right]\right|_{\xi=\xi_{1}} & \left.M\left[\mathcal{B}_{c}\right]\right|_{\xi=\xi_{1}} & \left.M\left[\mathcal{B}_{d}\right]\right|_{\xi=\xi_{1}} \\
\left.V\left[\mathcal{B}_{a}\right]\right|_{\xi=\xi_{1}} & \left.V\left[\mathcal{B}_{b}\right]\right|_{\xi=\xi_{1}} & \left.V\left[\mathcal{B}_{c}\right]\right|_{\xi=\xi_{1}} & \left.V\left[\mathcal{B}_{d}\right]\right|_{\xi=\xi_{1}} \\
\left.\mathcal{B}_{a}\right|_{\xi=1} & \left.\mathcal{B}_{b}\right|_{\xi=1} & \left.\mathcal{B}_{c}\right|_{\xi=1} & \left.\mathcal{B}_{d}\right|_{\xi=1} \\
\left.\frac{\partial \mathcal{B}_{a}}{\partial \xi}\right|_{\xi=1} & \left.\frac{\partial \mathcal{B}_{b}}{\partial \xi}\right|_{\xi=1} & \left.\frac{\partial \mathcal{B}_{c}}{\partial \xi}\right|_{\xi=1} & \left.\frac{\partial \mathcal{B}_{d}}{\partial \xi}\right|_{\xi=1}
\end{array}\right|
$$

— elastic constraints at the inner edge and free outer edge

$$
\Delta(\lambda, \boldsymbol{\kappa}, \mathbf{K}, \phi, \psi) \equiv\left|\begin{array}{llll}
\left.M\left[\mathcal{B}_{a}\right]\right|_{\xi=1} & \left.M\left[\mathcal{B}_{b}\right]\right|_{\xi=1} & \left.M\left[\mathcal{B}_{c}\right]\right|_{\xi=1} & \left.M\left[\mathcal{B}_{d}\right]\right|_{\xi=1} \\
\left.V\left[\mathcal{B}_{a}\right]\right|_{\xi=1} & \left.V\left[\mathcal{B}_{b}\right]\right|_{\xi=1} & \left.V\left[\mathcal{B}_{c}\right]\right|_{\xi=1} & \left.V\left[\mathcal{B}_{d}\right]\right|_{\xi=1} \\
\left.\Phi\left[\mathcal{B}_{a}\right]\right|_{\xi=\xi_{1}} & \left.\Phi\left[\mathcal{B}_{b}\right]\right|_{\xi=\xi_{1}} & \left.\Phi\left[\mathcal{B}_{c}\right]\right|_{\xi=\xi_{1}} & \left.\Phi\left[\mathcal{B}_{d}\right]\right|_{\xi=\xi_{1}} \\
\left.\Psi\left[\mathcal{B}_{a}\right]\right|_{\xi=\xi_{1}} & \left.\Psi\left[\mathcal{B}_{b}\right]\right|_{\xi=\xi_{1}} & \left.\Psi\left[\mathcal{B}_{c}\right]\right|_{\xi=\xi_{1}} & \left.\Psi\left[\mathcal{B}_{d}\right]\right|_{\xi=\xi_{1}}
\end{array}\right|
$$




\section{Results and discussion}

The numerical results for fundamental frequencies of elastically supported circular plates are presented in Tables 1 and 2 with comparison to the results by Azimi (1988), Ding (1994), Wang and Wang. (2003). The numerical results for fundamental frequencies of free vibration of free circular plates with rigid ring supports are presented in Table 3 with comparison to the results by Wang (2014). The numerical results for fundamental frequencies of free vibration of free elastically supported annular plates with different boundary condition at the inner edge are presented in Tables 4 and 5 for different combinations of the radius of the core and supports. The fundamental frequencies of free vibration of circular plates with elastic constraints and interior ring supports of variable stiffness are presented in Table 6. Additionally, the eigenvalues of circular plates with elastic constraints depending on radius and stiffness of interior ring supports are shown in Figs. 2 and 3.

Table 1. The fundamental frequency $\lambda_{0}$ of free vibration of circular plates with the elastic ring support

\begin{tabular}{|c|c|c|c|c|c|c|}
\hline \multirow[b]{2}{*}{$K_{1}$} & \multirow[b]{2}{*}{$\kappa_{1}$} & \multirow[b]{2}{*}{$\begin{array}{l}\text { Dimensionless } \\
\text { frequency } \lambda_{0}\end{array}$} & \multicolumn{4}{|c|}{ Boundary conditions } \\
\hline & & & Clamped & $\begin{array}{c}\text { Simply } \\
\text { supported }\end{array}$ & Free & Sliding \\
\hline \multirow{21}{*}{10} & 0 & GF & 3.196 & 2.221 & 0.211 & 0.212 \\
\hline & 0.1 & GF & 3.272 & 2.360 & 1.115 & 1.171 \\
\hline & \multirow{4}{*}{0.2} & GF & 3.326 & 2.460 & 1.357 & 1.383 \\
\hline & & Wang and Wang (2003) & 3.325 & 2.460 & - & - \\
\hline & & Azimi (1988) & 3.326 & 2.461 & - & - \\
\hline & & Ding (1994) & 3.322 & - & - & - \\
\hline & 0.3 & GF & 3.348 & 2.523 & 1.497 & 1.532 \\
\hline & \multirow{4}{*}{0.4} & GF & 3.338 & 2.547 & 1.620 & 1.656 \\
\hline & & Wang and Wang (2003) & 3.338 & 2.547 & - & - \\
\hline & & Azimi (1988) & 3.338 & 2.547 & - & - \\
\hline & & Ding (1994) & 3.334 & - & - & - \\
\hline & 0.5 & $\mathrm{GF}$ & 3.304 & 2.530 & 1.736 & 1.765 \\
\hline & \multirow{4}{*}{0.6} & GF & 3.262 & 2.478 & 1.844 & 1.856 \\
\hline & & Wang and Wang (2003) & 3.262 & 2.478 & - & - \\
\hline & & Azimi (1988) & 3.262 & 2.479 & - & - \\
\hline & & Ding (1994) & 3.262 & - & - & - \\
\hline & 0.7 & GF & 3.225 & 2.403 & 1.928 & 1.928 \\
\hline & \multirow{4}{*}{0.8} & GF & 3.204 & 2.321 & 1.960 & 1.980 \\
\hline & & Wang and Wang (2003) & 3.204 & 2.321 & 1.961 & $\overline{-}$ \\
\hline & & Azimi (1988) & 3.199 & 2.321 & - & - \\
\hline & & Ding (1994) & 3.204 & - & - & - \\
\hline
\end{tabular}

The fundamental frequencies of free vibration of annular plates with the clamped outer edge and the free inner edge (rigid interior support) are presented in Table 7 with comparison to the results by Vega (2000). The eigenvalues of free annular plates with elastic constraints at the inner edge and interior ring supports are presented in Table 8 for different combinations of the radius of the core and supports. 
Table 2. The fundamental frequency $\lambda_{0}$ of free vibration of circular plates with the elastic ring support

\begin{tabular}{|c|c|c|c|c|c|c|}
\hline \multirow[b]{2}{*}{$K_{1}$} & \multirow[b]{2}{*}{$\kappa_{1}$} & \multirow[b]{2}{*}{$\begin{array}{l}\text { Dimensionless } \\
\text { frequency } \lambda_{0}\end{array}$} & \multicolumn{4}{|c|}{ Boundary conditions } \\
\hline & & & Clamped & $\begin{array}{c}\text { Simply } \\
\text { supported }\end{array}$ & Free & Sliding \\
\hline \multirow{21}{*}{1000} & 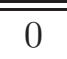 & $\overline{\mathrm{GF}}$ & 3.204 & 2.223 & 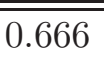 & $\overline{0.667}$ \\
\hline & 0.1 & GF & 4.677 & 3.805 & 1.946 & 2.238 \\
\hline & \multirow{4}{*}{0.2} & GF & 5.175 & 4.202 & 2.049 & 2.418 \\
\hline & & Wang and Wang (2003) & 5.175 & 4.202 & - & - \\
\hline & & Azimi (1988) & 5.187 & 4.210 & - & - \\
\hline & & Ding (1994) & 4.929 & - & - & - \\
\hline & 0.3 & GF & 5.763 & 4.682 & 2.187 & 2.656 \\
\hline & \multirow{4}{*}{0.4} & GF & 6.110 & 5.276 & 2.374 & 2.979 \\
\hline & & Wang and Wang (2003) & 6.110 & 5.276 & - & - \\
\hline & & Azimi (1988) & 6.129 & 5.282 & - & - \\
\hline & & Ding (1994) & 6.114 & - & - & - \\
\hline & 0.5 & GF & 5.195 & 5.136 & 2.619 & 3.403 \\
\hline & \multirow{4}{*}{0.6} & GF & 4.503 & 4.479 & 2.891 & 3.803 \\
\hline & & Wang and Wang (2003) & 4.503 & 4.479 & - & - \\
\hline & & Azimi (1988) & 4.512 & 4.486 & - & $\overline{-}$ \\
\hline & & Ding (1994) & 4.492 & - & - & - \\
\hline & 0.7 & GF & 3.967 & 3.962 & 2.992 & 3.707 \\
\hline & \multirow{4}{*}{0.8} & GF & 3.539 & 3.532 & 2.787 & 3.438 \\
\hline & & Wang and Wang (2003) & 3.539 & 3.532 & - & - \\
\hline & & Azimi (1988) & 3.547 & 3.537 & - & - \\
\hline & & Ding (1994) & 3.547 & - & - & - \\
\hline
\end{tabular}

Table 3. The fundamental frequency $\lambda_{0}$ of free vibration of free circular plates with the rigid ring support

\begin{tabular}{|c|c|c|c|}
\hline \multirow{2}{*}{$K_{1}$} & \multirow{2}{*}{$\kappa_{1}$} & \multicolumn{2}{|c|}{ Dimensionless frequency $\lambda_{0}$} \\
\cline { 3 - 4 } & & GF & Wang (2014) \\
\hline \hline \multirow{6}{*}{$\infty$} & 0 & 3.751 & 3.752 \\
\cline { 2 - 4 } & 0.1 & 3.909 & 3.909 \\
\hline & 0.2 & 4.275 & 4.275 \\
\cline { 2 - 4 } & 0.3 & 4.851 & 4.851 \\
\cline { 2 - 4 } & 0.4 & 5.706 & 5.707 \\
\cline { 2 - 4 } & 0.5 & 6.929 & 6.929 \\
\cline { 2 - 4 } & 0.6 & 8.396 & 8.390 \\
\cline { 2 - 4 } & 0.7 & 8.960 & 8.959 \\
\cline { 2 - 4 } & 0.8 & 7.809 & 7.809 \\
\cline { 2 - 4 } & 0.9 & 6.235 & 6.235 \\
\cline { 2 - 4 } & 1.0 & 4.935 & 4.935 \\
\hline
\end{tabular}


Table 4. The fundamental frequency $\lambda_{0}$ of free vibration of free annular plates with different boundary conditions at the inner edge and interior elastic support

\begin{tabular}{|c|c|c|c|c|c|c|c|}
\hline \multirow[b]{2}{*}{$K_{1}$} & \multirow[b]{2}{*}{$\xi_{1}$} & \multirow[b]{2}{*}{$\kappa_{1}$} & \multirow{2}{*}{$\begin{array}{l}\text { Dimensionless } \\
\text { frequency }\end{array}$} & \multicolumn{4}{|c|}{ Boundary conditions at the inner edge } \\
\hline & & & & Clamped & $\begin{array}{c}\text { Simply } \\
\text { supported }\end{array}$ & Free & Sliding \\
\hline \multirow{12}{*}{10} & 0.1 & 0.2 & \multirow{12}{*}{$\lambda_{0}$} & 2.043 & 1.826 & 1.350 & 1.364 \\
\hline & 0.1 & 0.4 & & 2.050 & 1.886 & 1.617 & 1.624 \\
\hline & 0.1 & 0.6 & & 2.207 & 2.091 & 1.848 & 1.848 \\
\hline & 0.1 & 0.9 & & 2.658 & 2.537 & 1.931 & 1.946 \\
\hline & 0.3 & 0.5 & & 2.569 & 1.918 & 1.730 & 1.778 \\
\hline & 0.3 & 0.7 & & 2.697 & 2.209 & 1.975 & 1.975 \\
\hline & 0.3 & 0.9 & & 3.003 & 2.587 & 1.959 & 2.041 \\
\hline & 0.5 & 0.7 & & 3.616 & 2.235 & 2.039 & 2.068 \\
\hline & 0.5 & 0.9 & & 3.812 & 2.736 & 2.086 & 2.193 \\
\hline & 0.7 & 0.8 & & 6.077 & 2.634 & 2.311 & 2.363 \\
\hline & 0.7 & 0.9 & & 6.119 & 3.008 & 2.401 & 2.436 \\
\hline & 0.8 & 0.9 & & 9.197 & 3.275 & 2.658 & 2.658 \\
\hline
\end{tabular}

Table 5. The fundamental frequency $\lambda_{0}$ of free vibration of free annular plates with different boundary conditions at the inner edge and interior elastic support

\begin{tabular}{|c|c|c|c|c|c|c|c|}
\hline \multirow[b]{2}{*}{$K_{1}$} & \multirow[b]{2}{*}{$\xi_{1}$} & \multirow[b]{2}{*}{$\kappa_{1}$} & \multirow{2}{*}{$\begin{array}{l}\text { Dimensionless } \\
\text { frequency }\end{array}$} & \multicolumn{4}{|c|}{ Boundary conditions at the inner edge } \\
\hline & & & & Clamped & $\begin{array}{c}\text { Simply } \\
\text { supported }\end{array}$ & Free & Sliding \\
\hline \multirow{12}{*}{1000} & 0.1 & 0.2 & \multirow{12}{*}{$\lambda_{0}$} & 2.087 & 1.925 & 1.979 & 2.091 \\
\hline & 0.1 & $\overline{0.4}$ & & 2.117 & 1.105 & 2.331 & 2.400 \\
\hline & 0.1 & 0.6 & & 9.335 & 3.843 & 2.852 & 2.934 \\
\hline & 0.1 & 0.9 & & 4.685 & 4.215 & 2.467 & 2.545 \\
\hline & 0.3 & 0.5 & & 2.684 & 7.401 & 2.352 & 2.859 \\
\hline & 0.3 & 0.7 & & 12.106 & 4.769 & 2.887 & 3.561 \\
\hline & 0.3 & 0.9 & & 6.175 & 5.214 & 2.439 & 2.987 \\
\hline & 0.5 & 0.7 & & 13.526 & 13.323 & 2.859 & 4.266 \\
\hline & 0.5 & 0.9 & & 9.260 & 7.249 & 2.648 & 4.106 \\
\hline & 0.7 & 0.8 & & 5.526 & 6.437 & 3.293 & 6.321 \\
\hline & 0.7 & 0.9 & & 8.396 & 8.054 & 3.432 & 7.049 \\
\hline & 0.8 & 0.9 & & 9.697 & 7.774 & 4.294 & 8.334 \\
\hline
\end{tabular}




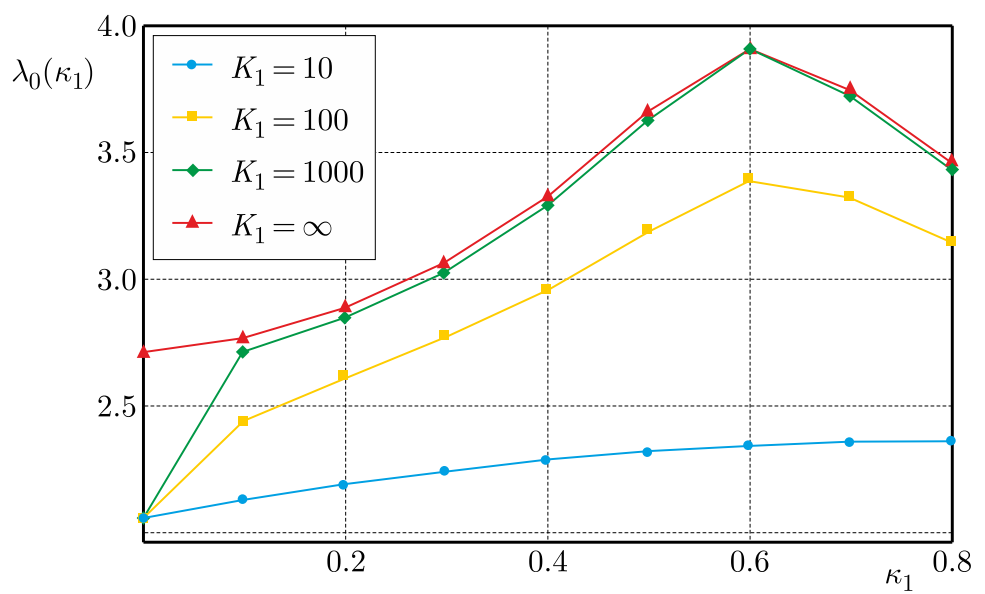

Fig. 2. The fundamental frequency of the circular plate with elastic constraints $(\phi=100, \psi=10)$ depending on the radius and stiffness of interior ring supports

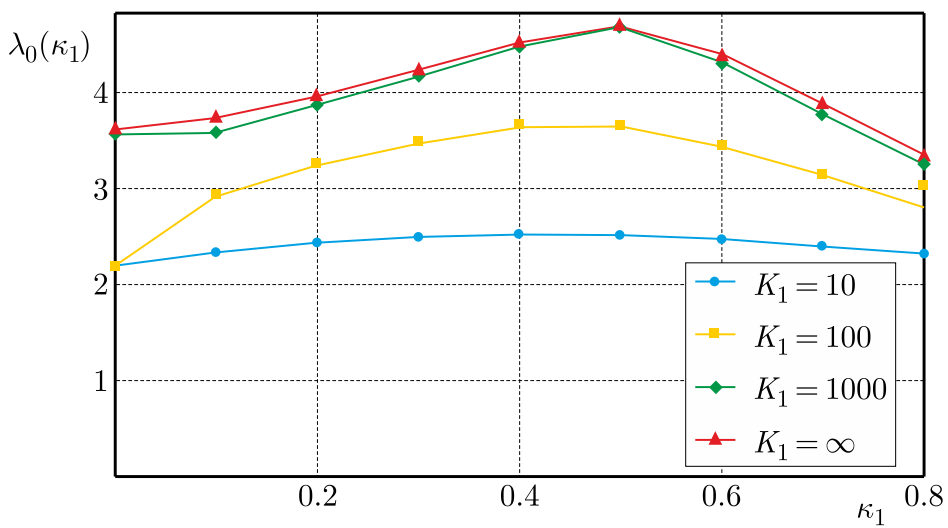

Fig. 3. The fundamental frequency of the circular plate with elastic constraints $(\phi=0.1, \psi=100)$ depending on the radius and stiffness of interior ring supports

Table 7. The fundamental frequency $\lambda_{0}$ of free vibration of annular plates with the clamped outer edge and free inner edge and the rigid interior support $\left(K_{1} \rightarrow \infty\right)$

\begin{tabular}{|c|c|c|c|c|c|c|c|c|c|}
\hline \multirow{3}{*}{$\xi_{1}$} & \multicolumn{9}{|c|}{$\kappa_{1}$} \\
\hline & & $\overline{0.2}$ & $\overline{0.3}$ & 0.4 & 0.5 & $\overline{0.6}$ & 0.7 & 0.8 & 0.9 \\
\hline & \multicolumn{9}{|c|}{ Dimensionless frequency $\lambda_{0}$} \\
\hline \multirow{2}{*}{0.1} & $\overline{\mathrm{GF}}$ & 5.335 & 5.946 & 6.262 & 5.537 & 4.782 & 4.215 & 3.786 & 3.453 \\
\hline & Vega et al. (2000) & 5.335 & 5.946 & 6.262 & 5.537 & 4.782 & 4.215 & 3.786 & 3.453 \\
\hline \multirow{2}{*}{0.2} & $\overline{\mathrm{GF}}$ & & 5.890 & 6.530 & 5.996 & 5.051 & 4.370 & 3.880 & 3.512 \\
\hline & Vega et al. $(2000)$ & & 5.890 & 6.530 & 5.996 & 5.051 & 4.370 & 3.880 & 3.512 \\
\hline \multirow{2}{*}{0.3} & $\overline{\mathrm{GF}}$ & & & 6.723 & 7.100 & 5.853 & 4.856 & 4.194 & 3.727 \\
\hline & Vega et al. (2000) & & & 6.723 & 7.100 & 5.853 & 4.856 & 4.194 & 3.727 \\
\hline \multirow{2}{*}{0.4} & GF & & & & 7.912 & 7.527 & 5.856 & 4.821 & 4.154 \\
\hline & Vega et al. (2000) & & & & 7.912 & 7.527 & 5.856 & 4.821 & 4.154 \\
\hline \multirow{2}{*}{0.5} & $\overline{\mathrm{GF}}$ & & & & & 9.612 & 7.874 & 5.969 & 4.894 \\
\hline & Vega et al. (2000) & & & & & 9.612 & 7.874 & 5.969 & 4.894 \\
\hline \multirow{2}{*}{0.6} & $\overline{G F}$ & & & & & & 11.912 & 8.256 & 6.195 \\
\hline & Vega et al. (2000) & & & & & & 11.912 & 8.256 & 6.196 \\
\hline \multirow{2}{*}{0.7} & GF & & & & & & & 14.146 & 8.785 \\
\hline & Vega et al. (2000) & & & & & & & 14.147 & 8.785 \\
\hline 0.8 & $\overline{\mathrm{GF}}$ & & & & & & & & 15.991 \\
\hline
\end{tabular}


Table 6. The fundamental frequency $\lambda_{0}$ of free vibration of circular plates with elastic constraints and the interior ring support

\begin{tabular}{|c|c|c|c|c|}
\hline \multirow{2}{*}{$K_{1}$} & \multirow{2}{*}{$\kappa_{1}$} & \multirow{2}{*}{$\begin{array}{c}\text { Dimensionless } \\
\text { frequency }\end{array}$} & \multicolumn{2}{|c|}{ Elastic parameters at the outer edge } \\
\hline & & & $\phi=100, \psi=10$ & $\phi=0.1, \psi=100$ \\
\hline \multirow{9}{*}{10} & $\overline{0}$ & \multirow{9}{*}{$\lambda_{0}$} & 2.056 & 2.203 \\
\hline & 0.1 & & 2.129 & 2.337 \\
\hline & 0.2 & & 2.190 & 2.434 \\
\hline & 0.3 & & 2.241 & 2.497 \\
\hline & 0.4 & & 2.285 & 2.524 \\
\hline & 0.5 & & 2.320 & 2.513 \\
\hline & 0.6 & & 2.344 & 2.469 \\
\hline & 0.7 & & 2.357 & 2.400 \\
\hline & 0.8 & & 2.361 & 2.321 \\
\hline \multirow{9}{*}{100} & 0 & \multirow{9}{*}{$\lambda_{0}$} & 2.056 & 2.203 \\
\hline & 0.1 & & 2.441 & 2.928 \\
\hline & 0.2 & & 2.610 & 3.247 \\
\hline & 0.3 & & 2.770 & 3.486 \\
\hline & 0.4 & & 2.960 & 3.650 \\
\hline & 0.5 & & 3.188 & 3.648 \\
\hline & 0.6 & & 3.387 & 3.445 \\
\hline & 0.7 & & 3.318 & 3.145 \\
\hline & 0.8 & & 3.142 & 2.809 \\
\hline \multirow{9}{*}{1000} & 0 & \multirow{9}{*}{$\lambda_{0}$} & 2.056 & 3.563 \\
\hline & 0.1 & & 2.715 & 3.600 \\
\hline & 0.2 & & 2.850 & 3.878 \\
\hline & 0.3 & & 3.032 & 4.176 \\
\hline & 0.4 & & 3.287 & 4.490 \\
\hline & 0.5 & & 3.629 & 4.700 \\
\hline & 0.6 & & 3.907 & 4.321 \\
\hline & 0.7 & & 3.719 & 3.786 \\
\hline & 0.8 & & 3.429 & 3.263 \\
\hline \multirow{9}{*}{$\infty$} & 0 & \multirow{9}{*}{$\lambda_{0}$} & 2.714 & 3.624 \\
\hline & 0.1 & & 2.769 & 3.746 \\
\hline & 0.2 & & 2.888 & 3.973 \\
\hline & 0.3 & & 3.068 & 4.250 \\
\hline & 0.4 & & 3.327 & 4.536 \\
\hline & 0.5 & & 3.664 & 4.700 \\
\hline & 0.6 & & 3.908 & 4.410 \\
\hline & 0.7 & & 3.743 & 3.892 \\
\hline & 0.8 & & 3.458 & 3.354 \\
\hline
\end{tabular}

The Poisson ratio is taken as $\nu=0.3$ for all considered cases. The numerical results are obtained by using the Newton-Raphson method and Mathematica v10 software. The obtained results are in good agreement with the results obtained by other methods presented in literature and can be used to validate the accuracy of other numerical methods as benchmark values. 
Table 8. The fundamental frequency $\lambda_{0}$ of free vibration of free annular plates with elastic constraints at the inner edge and interior ring support

\begin{tabular}{|c|c|c|c|c|c|}
\hline \multirow{2}{*}{$K_{1}$} & \multirow{2}{*}{$\xi_{1}$} & \multirow{2}{*}{$\kappa_{1}$} & \multirow{2}{*}{$\begin{array}{c}\text { Dimensionless } \\
\text { frequency }\end{array}$} & \multicolumn{2}{|c|}{ Elastic parameters at the inner edge } \\
\hline & & & & $\phi=100, \psi=10$ & $\phi=0.1, \psi=100$ \\
\hline \multirow{12}{*}{10} & $\overline{0.1}$ & $\overline{0.2}$ & \multirow{12}{*}{$\lambda_{0}$} & $\overline{1.146}$ & $\overline{1.556}$ \\
\hline & 0.1 & 0.4 & & 1.530 & 1.740 \\
\hline & 0.1 & 0.6 & & 1.814 & 2.001 \\
\hline & 0.1 & 0.9 & & 2.031 & 2.439 \\
\hline & 0.3 & 0.5 & & 1.537 & 1.956 \\
\hline & 0.3 & 0.7 & & 1.839 & 2.238 \\
\hline & 0.3 & $\overline{0.9}$ & & 1.974 & 2.625 \\
\hline & 0.5 & 0.7 & & 1.648 & 2.296 \\
\hline & 0.5 & 0.9 & & 1.894 & 2.788 \\
\hline & 0.7 & $\overline{0.8}$ & & 1.510 & 2.680 \\
\hline & 0.7 & 0.9 & & 1.759 & 3.050 \\
\hline & 0.8 & $\overline{0.9}$ & & 1.603 & 3.237 \\
\hline \multirow{12}{*}{1000} & 0.1 & 0.2 & \multirow{12}{*}{$\lambda_{0}$} & 2.101 & 1.941 \\
\hline & 0.1 & 0.4 & & 2.460 & 6.152 \\
\hline & 0.1 & 0.6 & & 3.091 & 3.663 \\
\hline & 0.1 & 0.9 & & 2.855 & 4.239 \\
\hline & 0.3 & 0.5 & & 2.906 & 0.852 \\
\hline & 0.3 & 0.7 & & 3.739 & 4.951 \\
\hline & 0.3 & $\overline{0.9}$ & & 3.212 & 6.150 \\
\hline & 0.5 & 0.7 & & 4.332 & 6.707 \\
\hline & 0.5 & 0.9 & & 4.267 & 7.911 \\
\hline & 0.7 & 0.8 & & 6.374 & 7.977 \\
\hline & 0.7 & 0.9 & & 7.185 & 8.053 \\
\hline & 0.8 & 0.9 & & 8.319 & 8.196 \\
\hline \multirow{12}{*}{$\infty$} & 0.1 & 0.2 & \multirow{12}{*}{$\lambda_{0}$} & 3.308 & 1.296 \\
\hline & 0.1 & $\overline{0.4}$ & & 3.687 & 1.463 \\
\hline & 0.1 & 0.6 & & 3.972 & 2.000 \\
\hline & 0.1 & 0.9 & & 3.022 & 1.351 \\
\hline & 0.3 & 0.5 & & 1.175 & 1.937 \\
\hline & 0.3 & 0.7 & & 2.071 & 2.120 \\
\hline & 0.3 & 0.9 & & 3.834 & 1.271 \\
\hline & 0.5 & 0.7 & & 2.995 & 2.030 \\
\hline & 0.5 & 0.9 & & 2.644 & 1.335 \\
\hline & 0.7 & 0.8 & & 1.998 & 0.999 \\
\hline & 0.7 & 0.9 & & 1.683 & 1.385 \\
\hline & 0.8 & 0.9 & & 1.046 & 1.293 \\
\hline
\end{tabular}

\section{Conclusions}

In this paper, the quasi-Green function has been employed to solve natural vibration of elastically supported thin circular and annular plates with different boundary conditions. The advantage of quasi-Green's function is the obtaining of characteristic equations without calculating nontrivial constants in complicated forms. Additionally, the number of supports of circular and annular 
plates does not influence the dimension of characteristic matrices, because the continuity conditions can be neglected. In the presented approach, the solution to the boundary value problem is much simpler. The quasi-Green function approach can be used to the frequency analysis of plates and beams with other discrete elements such as an additional mass or a mass on the spring. The exact frequencies of vibration presented in a non-dimensional form can serve as benchmark values for researchers to validate their numerical methods applied in similar problems presented in the paper.

\section{Acknowledgements}

The research was conducted within S/WZ/1/2014 project and was financed by the funds of the Ministry of Science and Higher Education, Poland.

\section{References}

1. AzImi S., 1988, Free vibration of circular plates with elastic or rigid interior support, Journal of Sound and Vibration, 120, 37-53

2. Bodine R.Y., 1967, Vibration of a circular plate supported by a concentric ring of arbitrary radius, Journal of the Acoustical Society of America, 41, 1551

3. Ding Z., 1994, Free vibration of arbitrarily shaped plates with concentric ring elastic and rigid supports, Computers and Structures, 50, 685-692

4. Jaroszewicz J., Zoryj L., 2005, Methods of Free Axisymmetric Vibration Analysis of Circular Plates Using by Influence Functions, Bialystok University of Technology, Poland

5. Kukla S., Szewczyk M., 2004, The Green's functions for vibration problems of circular plates with elastic ring supports, Scientific Research of the Institute of Mathematics and Computer Science, 4, 1, 79-86

6. Kukla S., Szewczyk M., 2005, Application of Green's function method in frequency analysis of axisymmetric vibration of annular plates with elastic ring supports, Scientific Research of the Institute of Mathematics and Computer Science, 3, 1, 67-72

7. Kukla S., Szewczyk M., 2007, Frequency analysis of annular plates with elastic concentric supports by Green's function method, Journal of Sound and Vibration, 300, 387-393

8. KunukKasseril V.X., Swamidas A.S.J., 1974, Vibration of continuous circular plates, International Journal of Solids and Structures, 10,603-619

9. Laura P.A.A., Gutierrez R.H., Vera S.A., Vega D.A., 1999, Transverse vibrations of circular plate with a free edge and a concentric circular support, Journal of Sound and Vibration, 223, $5,843-845$

10. LiU C.F., Chen G.T., 1995, A simple finite element analysis of axisymmetric vibration of annular and circular plates, International Journal of Mechanical Sciences, 37, 8, 861-871

11. McLachlan N.W., 1955, Bessel Functions for Engineers, Clarendon Press, Oxford

12. RAO L.B., RAO C.K., 2014a, Frequencies of circular plate with concentric ring and elastic edge support, Frontiers on Mechanical Engineering, 9, 2, 168-176

13. RaO L.B., RAO C.K., 2014b, Frequency analysis of annular plates with inner and outer edges elastically restrained and resting on Winkler foundation, International Journal of Mechanical Sciences, 81, 184-194

14. Singh A.V., Mirza S., 1976, Free axisymmetric vibration of a circular plate elastically supported along two concentric circles, Journal of Sound and Vibration, 48, 425-429

15. Vega D.A., Vera S.A., Laura P.A.A., Gutierrez R.H., Pronsato M.E., 1999, Transverse vibrations of an annular circular plate with free edges and an intermediate concentric circular support, Journal of Sound and Vibration, 223, 493-496 
16. Vega D.A., Laura P.A.A., Vera S.A., 2000, Vibrations of an annular isotropic plate with one edge clamped or simply supported and an intermediate concentric circular supports, Journal of Sound and Vibration, 233, 1, 171-174

17. Wang C.M., Thevendran V., 1993, Vibration analysis of annular plates with concentric supports using a variant of Rayleigh-Ritz method, Journal of Sound and Vibration, 163, 137-149

18. WANG C.Y., 2001, On the fundamental frequency of a circular plate supported on a ring, Journal of Sound and Vibration, 243, 5, 945-946

19. WAng C.Y., WAng C.M., 2003, Fundamental frequencies of circular plates with internal elastic ring support, Journal of Sound and Vibration, 263, 1071-1078

20. WAng C.Y., 2006, Fundamental frequencies of annular plates with movable edges, Journal of Sound and Vibration, 290, 524-528

21. WANG C.Y., 2014, The vibration modes of concentrically supported free circular plates, Journal of Sound and Vibration, 333, 835-847

22. ŻUR K.K., 2015, Green's function in frequency analysis of circular thin plates of variable thickness, Journal of Theoretical and Applied Mechanics, 53, 4, 873-884

23. ŻUR K.K., 2016a, Green's function approach to frequency analysis of thin circular plates, Bulletin of the Polish Academy of Sciences - Technical Sciences, 64, 1, 181-188

24. ZUR K.K., 2016b, Green's function for frequency analysis of thin annular plates with nonlinear variable thickness, Applied Mathematical Modelling, 40, 5-6, 3601-3619 